\title{
A Proposal for Masonry Bridge Health Assessment Using Al and Semantics
}

\author{
Raissa Garozzo
}

\section{Abstract}

Masonry railway bridges represent a historical built heritage to be preserved. This contribution proposes a new methodological approach for health assessment of masonry railway bridges based on the definition of image-based and Al-driven survey protocols useful for the creation of semi-automated $\mathrm{H}-\mathrm{BIM}$ models. To do this, a heuristic approach is required with the integrated combination of techniques and methodologies belonging to different fields. As case studies the masonry bridges of the sicilian Circumetnea railway are chosen.

Keywords

railway masonry arch bridge, digital survey, archival research, artificial intelligence, GANs.
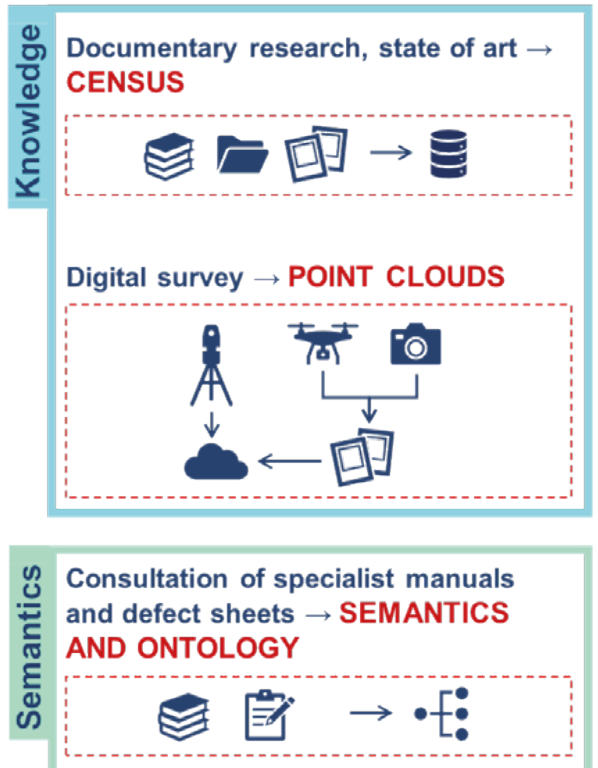

$\bar{\varangle}$

DNN training using Ontology-GANs $\rightarrow$ AUTOMATIC POINT CLOUD SEGMENTATION

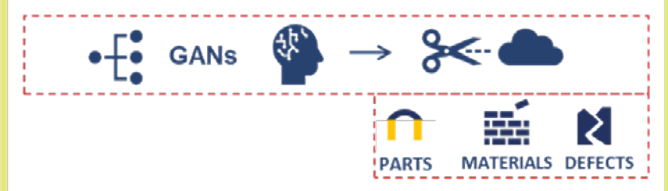

$\underline{M}$
I

Identification of parametrizable characters and creation of parametric components $\rightarrow$ H-BIM MODELS

$$
\text { •f: }
$$

Analysis of interoperability issues 


\section{Introduction}

Masonry arch bridges are one of the most common structural typologies in the worldwide architectural heritage. In the nineteenth century, the design and construction of bridges had a great impulse with the railways' advent. While the function of the bridges has changed over time, due to the abandoned railway networks, their value as historical permanence of the past has increased over time, even enhancing the value of the landscape.

The architectural qualities of these assets make them testimonies of past theoretical knowledge and construction skills; the natural obsolescence threatens this key heritage due to neglect and lack of maintenance in the case of disused bridges. Therefore, a remarkable number of bridges have collapsed or failed worldwide over the past years. The stability and safety of still-in-use masonry bridges must be guaranteed, avoiding over-dimensioned intervention and, in the worst-case scenarios, demolition and reconstruction.

Hence the need for a strategy of expeditious health monitoring of masonry arch bridges, aimed at containing the potential damages caused by seismic, hydrogeological, and other vulnerability assessments. It is crucial to develop effective and integrated procedures to characterize the structural conditions, identify and prevent potential vulnerabilities of such historic assets studying their geometric configurations, construction techniques, and documentary heritage. In this direction the current Italian guides for risk classification and management, safety assessment and monitoring of existing bridges give pivotal suggestions.

$\mathrm{H}-\mathrm{BIM}$ approach could be the starting point for management, conservation, and maintenance of these historical assets, as it gathers all present and past information on the artefact. Besides that, $\mathrm{H}-\mathrm{BIM}$ could be used throughout the life cycle of a bridge, as a management supporting tool constantly updated. The knowledge base of such an information model is the point cloud, acquired with different methodologies depending on the characteristics of the case study.

Despite the tremendous advantages of this technology, one of the principal limitations is the time and training required to manually process the huge quantity of data that point clouds contain. In this regard, Artificial Intelligence (Al) techniques and semantic web could really help in the process. For example, ontologies could serve in helping neural networks in semi-automatic point cloud segmentation or adding semantic layers to $\mathrm{H}-\mathrm{BIM}$ models. Also, the bridge's state of health is commonly assessed by visual inspection, and this is a time consuming, expensive, and laborious procedure.

Computer vision with Deep Learning $(\mathrm{DL})$ techniques can support professional users in damage detection and classification. However, Al methods, based on the DL paradigm, require a significant amount of annotated data that, in this context, it is often impossible to collect. To make up for this issue it is possible to exploit data augmenting techniques to create synthetic data to enrich the dataset, through an approach based on Generative Adversarial Networks (GANs).

In this regard, this manuscript aims at proposing a new methodological approach to define imagebased and Al-driven survey protocols useful for the creation of semi-automated $\mathrm{H}$-BIM models for health assessment of masonry railway bridges.

To support the research purposes, the chosen case studies are the masonry bridges of Circumetnea, a still-in-service railway that almost encircles the Etna Volcano, passing through several towns in Etna's foothills.

\section{State of Art}

Automating the point cloud-to-Bridge Information Models (BIM) process can significantly reduce the effort and cost in masonry bridge inspections and management.

Recently, Zhao and Vela [2019] provides a pipeline for simple concrete bridges scan-to$\mathrm{BIM}$, integrating several procedures related to segmentation, surface model estimation, and classification of surface regions. Also Xu and Turkan [2019] underlines the need to identify productive approaches to inspect and manage bridges, proposing a framework that integrates BIM and Unmanned Aerial System Services (UASs) technologies. In León-Robles et al. [2019], historical-archival research and digital survey are used as a database to create a Historical $(H) B I M$. 
The automatic or semi-automatic point cloud segmentation can considerably support information modelling. Kim et al. [2020] present a methodology for the automated concrete bridge component recognition using deep learning, while Truong-Hong and Lindenbergh [202I] introduce an approach to automatically extract the point cloud of each surface of structural components of a slab bridge. The researchers are showing an increasing interest in an effective pipeline for masonry bridges as well. In Riveiro et al. [20।6], a method for the automatic segmentation of historic masonry bridges was achieved based on the combination of a heuristic approach and image processing tools adapted to voxel structures. Using these results, a methodology to automatically transform classified point clouds into IFC models for further applications is proposed by Sánchez Rodríguez et al. [2020].

Given this, a BIM approach supported by automatic and semi-automatic point cloud segmentation can help protect, managing, and enhancing historically rich assets, such as masonry arch bridges [Savini et al. 202 I].

\section{Methodology}

The methodology proposed is structured as it follows:

Cognitive phase, aimed at knowing and documenting the case studies, was developed according to the following steps:

- Documentary research - it allows the understanding of the design idea and provides important indications on the artefact, especially regarding the geometric configuration and the relationship with the pre-existing structures at the time of construction;

- Integrated digital survey campaigns - it is aimed at acquiring the geometrical and material configuration of the bridges and is carried out by using TLS, SFM photogrammetry and the experimentation and validation of videogrammetry techniques;

- Census - it brings together all the information acquired during the cognitive phase in a geographical information system, allowing to understand the bridges analysed relation with the entire railway and the territory.

Semantic phase, aimed at designing a masonry arched bridge computational ontology that will be used as a knowledge-base for deep neural network training, needed of:

- Conceptualisation - it provides an in-depth survey of existing vocabularies and taxonomies. Then, identifying relationships and hierarchies between parts to choose the proper classes, subclasses, and property of the developing ontology is required. To do this it is crucial to conduct in-depth research into technical manuals and treatises, as it is analysing several case studies and their typologies, as reported in.

- Comparison of existing ontologies - it is useful to understand whether it is better to link to something existing or create a new ontology.

- Ontology development - for which a particular attention is given to the level of granularity, if it is as an extension from existing standards. Some levels of information to be added, for instance, are related to the semantic structure, construction techniques, and typical defects.

- Test phase - in which the developed ontology is used for training a deep neural network. In doing this, an attempt is made to understand if the chosen ontology standard fits well with the research purposes.

Artificial intelligence (Al) approach, which aims to train a neural network for point cloud segmentation and visual-based defect detection, both on the numerical models and images.

- Creation of a two-level GAN - it is composed by a first GAN that generates isolated objects corresponding to each ontology concept, and a second one that combines the generated objects according to the spatial information provided by the ontology, to generate realistic scenes. To create a synthetic dataset, it is necessary to start from real images. For this reason, a massive acquisition of pictures of the same typology as the case study is necessary.

- Training of the DNN with real and previously mentioned synthetic datasets

- Testing phase on existing bridges point clouds

Information Modelling phase, aimed at creating parametric components, starting from the segmented point clouds. 


\section{First Results}

The case study chosen for the proposed experimentation is the Circumetnea railway masonry arch bridges. Built between 1889 and I 895, the Circumetnea is a regional narrow-gauge railway that connects Catania to Riposto, almost encircling the volcano Etna and passing through several towns in the foothills of Etna. The heterogeneity of the typologies (number of arches, materials, geometry) and the recurrence of the types, which include many instances, made them the ideal case study for this research. Finally, it is a heritage at risk, because some of the bridges have recently been demolished because of changes in traffic requirements.

Cognitive phase - The first step in the cognitive phase was an in-depth documentary research carried out at the State Archive of Catania, which holds 192 folders of original drawings and documents dated back to the timing of construction of Circumetnea. Plans and longitudinal profiles of the rail route, together with bridge projects and metric computations, were acquired digitally. This documentation allows the investigation of unknown construction and technological features, such as foundation typologies, and a better understanding of the reasons and the developments of the project. The documentary research was coupled with the digital survey campaign. The integration of several surveying methodologies, such as laser scanning, photogrammetry (ground and drone-assisted) and videogrammetry, was required due to the peculiarities of the analysis. A total of 37 bridges were identified along the route under investigation. Only 13 of these were accessible. 18 of them were photographed and only II were surveyed, using the above mentioned techniques (fig. I). The census was conducted concomitantly with the previous two stages. The bridges were first detected using Google Earth and then loaded into QGIS, in order to produce a grid of attributes organised according to the position (latitude and longitude), number of arches, building materials, archival documentation and survey operations that were carried out.

Fig. I. The result of the survey campain for a three-arch bridge in the municipality of Mascali (CT). The instruments used are: a NIKON D5300 (photogrammetry), Leica BLK360 (TLS), D MAVIC 2 PRO (UAV photogrammetry)
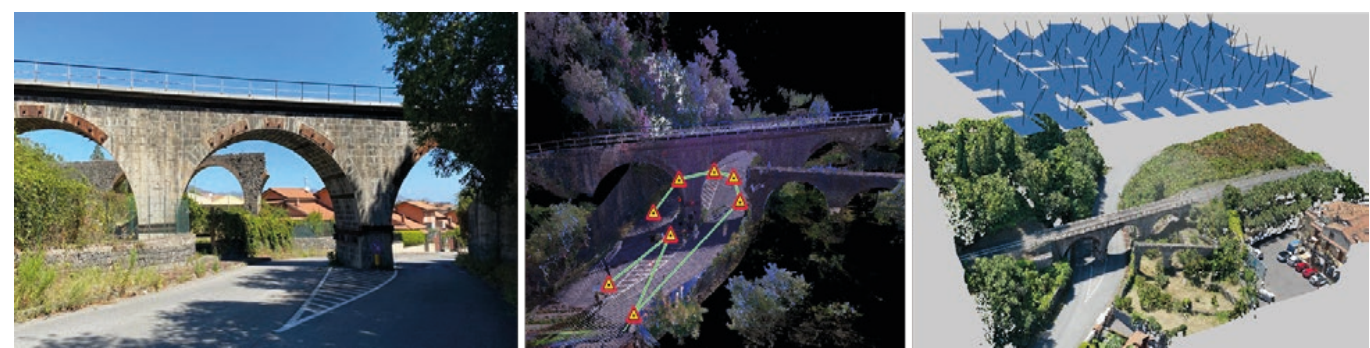

Semantic phase - The creation of a specific masonry bridge ontology started with the conceptualization phase. At the first stage, a multifaceted bottom-up analysis of the elements constituting this architectural typology, referring to well-defined thesauri and taxonomies, such as the AAT (Getty Research Institute) was conducted.

The actual conceptualization phase then began, based on the comprehensive text [Torre 2003], which helped in the semantic organisation. The element composing bridges are included in Physical Object, that could have Physical Property, such as materials, shape, and defects. Classes, as well as materials and shape, were obtained consulting manuals and historical documentation and through the case study observation. For defects, some inspection sheets previously produced for the Circumetnea under an agreement with our Department were consulted (fig. 2). Here, it moved to the comparison among existing ontologies; the analysis was conducted on CIDOC, ISO 2 I I 27 standard, which is the fundamental ontology for the management of cultural heritage information, and on IFC, ISO 16739 standard, open format for the management of interoperability.

Tothe author knowledge, there are currently no CIDOC extensions on masonry bridges. It is, therefore, interesting to explore the opportunities on such a diverse asset, which require a multidisciplinary approach for the safeguard of our cultural heritage. Nevertheless, the IFC-bridge is currently a project under development; also, using a single standard ontology as IFC to manage the semantic of built heritage in multiple applications could be an exciting challenge that simplifies information management and potentially bridging the gap between digital surveying, information modelling, and Al applications. In the light of this, it was decided to extend the 'IFC bridge', acting on its granularity. 
Fig. 2. The

conceptualization schema (on the left); the detail of the defects developed part (on the right).

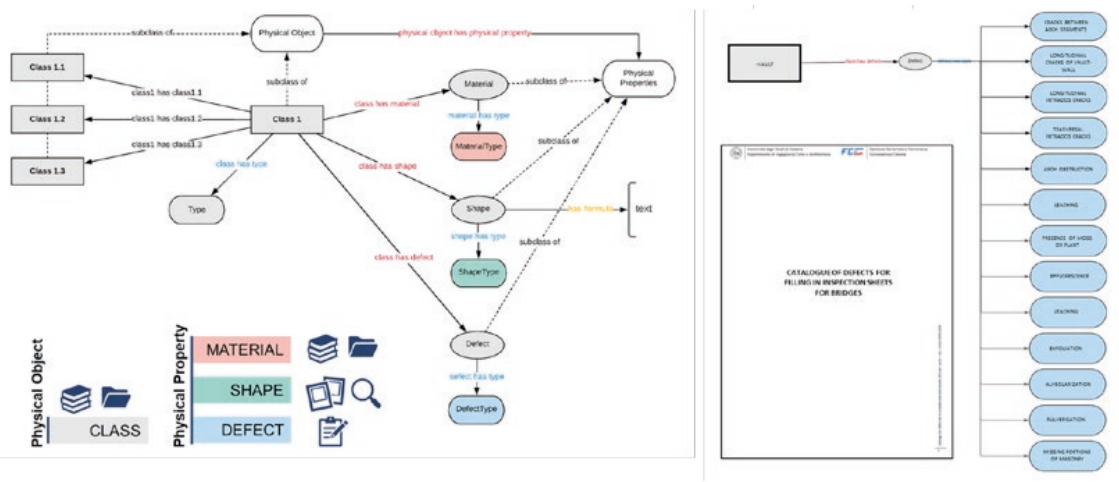

Al approach - At this early stage of the research, the necessary material for the creation of the synthetic dataset was acquired. A masonry arch bridge dataset was collected by the web, consisting of 10.446 images of 3.000 masonry arch bridges.

These data were built using a web scraping technique on Structurae.net, an international database and gallery of structures. Data collection was a key point in the training process, as a poorly built dataset could lead to bad performances or prevent the GAN model from learning. After images were scraped it took several days of manual data cleaning, erasing bad quality images, to obtain the final dataset used for the training. Images of aqueducts, drawings and plans, images with altered colouring, images that did not represent significant elements of bridges (such as statues and, more generally, decorative elements) were removed from the dataset. The images left after cleaning operations is a total of 7434 images.

\section{Conclusions and Future Development}

The results obtained at this stage of the research are encouraging, the conceptual formalization of the ontology added a new layer of knowledge to this valuable heritage. The experimentation will continue focusing on the integration of the ontology and, consequently, on the creation of the synthetic dataset and the segmentation of the point clouds. With regard to parametric modelling, an in-depth investigation will be carried out to identify a good pipeline to maintain a strong model fit with parameterisation of its components.

\section{References}

Kim Hyunjun, Yoon Jinyoung, Sim Sung-Han (2020). Automated bridge component recognition from point clouds using deep learning. In Structural Control and Health Monitoring, 27 (9), pp. I- I3.

León-Robles Carlos A., Reinoso-Gordo Juan F., González-Quiñones Juan J. (2019). Heritage Building Information Modeling (H-BIM) Applied to a Stone Bridge. In ISPRS International Journal of Geo-Information, 8 (3), I I I, pp. I-I4.

Riveiro Belen, Dejong Matthew J., Conde Borja (2016). Automated processing of large point clouds for structural health monitoring of masonry arch bridges. In Automation in Construction, 72, pp. 258-268.

Sánchez Rodríguez Ana, Esserb Sebastian, Abualdenienb Jimmy, Borrmannb Andre, Riveiro Belén (2020). From point cloud to IFC: A masonry arch bridge case study. In Ungureanu Lucian Constantin, Hartmann Timo (eds.). 27th International workshop on intelligent computing in engineering. Berlin: Universitätsverlag der TU Berlin.

Torre Camilla (2003). Ponti in muratura: dizionario storico-tecnologico. Firenze: Alinea.

Truong-Hong Linh, Lindenbergh Roderik (202I). Extracting Bridge Components from a Laser Scanning Point Cloud. In Toledo Santos Eduardo, Scheer Sergio (eds.). Proceedings of the 18th International Conference on Computing in Civil and Building Engineering. Cham: Springer International Publishing, pp. 721-739.

Xu Yiye, Turkan Yelda (2019). Bridge Inspection Using Bridge Information Modeling (BrIM) and Unmanned Aerial System (UAS). In Mutis Ivan, Hartmann, Timo (eds.). Advances in Informatics and Computing in Civil and Construction Engineering. Cham: Springer International Publishing, pp. 6 17-624.

Zhao Yi-Pu, Vela Patricio A. (2019). Scan2BrIM: IFC Model Generation of Concrete Bridges from Point Clouds. In Computing in Civil Engineering 2019. ASCE International Conference on Computing in Civil Engineering, Atlanta: American Society of Civil Engineers, pp. 455-463.

Author

Raissa Garozzo, Dept. of Civil Engineering and Architecture, University of Catania, raissa.garozzo@unict.it 
